\title{
MAXIMAL QUOTIENT RINGS AND ESSENTIAL RIGHT IDEALS IN GROUP RINGS OF LOCALLY FINITE GROUPS
}

\author{
Ferran Cedó* and Brian Hartzey
}

Dedicated to the memory of Pere Menal

\begin{abstract}
Let $k$ be a commutative field. Let $G$ be a locally finite group without elements of order $p$ in case char $k=p>0$. In this paper it is proved that the type $I_{\infty}$ part of the maximal right quotient ring of the group algebgra $k G$ is zero.
\end{abstract}

\section{Introduction}

Let $k$ be a commutative field, $G$ a group, and suppose that the group ring $k G$ is regular in the sense of von Neumann. By [7, p.69], this means precisely that $G$ is locally finite with no elements of order equal to the characteristic of $k$. Then the maximal right quotient ring $Q^{r}(k G)$ of $k G$ is a regular right self-injective ring [3, Corollaries 1.2 and 1.24$]$, and as such, is uniquely a direct product of rings of types $I_{f}, I_{\infty}, I I_{f}, I I_{\infty}$, and $I I I$. We refer to [3] for general background on regular rings. The main theorem of this paper is the following.

Theorem. With the above notation, the type $I_{\infty}$ part of $Q^{r}(k G)$ is zero.

The first author has obtained this result under various supplementary hypotheses [2], and in particular when $G$ is $\Delta$-hypercentral. These results will be used in the proof of the general case. He has also shown that the type $I_{f}$ part of $Q^{r}(k G)$ is non-zero if and only if $|G: \Delta(G)|<\infty$ and $\Delta(G)^{\prime}$ is finite, where as usual, $\Delta(G)$ is the subgroup of $G$ consisting of the elements with finitely many conjugates $[\boldsymbol{1}]$.

*Partially supported by the grant PB89-0296 of the CICYT. 
In 1983, Menal proposed the study of the maximal quotient ring of regular group algebras to the first author. One of the problems proposed was the characterization of the type I part of $Q^{r}(k G)$ for $k G$ regular. Goursaud and Valette [4] had a partial result, namely they had characterized this part when $k$ has positive characteristic or contains all roots of unity. Finally, with [1, Theorem 2.3] and the Theorem of this paper, the problem has been solved.

The remainder of the paper began as an attempt to answer the following question, raised for example in [6].

Question. Let $G$ be a locally finite group, $k$ a field and $H$ an infinite subgroup of $G$. Is it true that if $J$ is an essential right ideal of $k H$, then the right ideal $J k G$ of $k G$ generated by $J$ is essential in $k G$ ?

We are only able to answer this question in a very spccial case, namely the case when $J$ is the augmentation ideal of $k H$. However the method we use involves attaching a certain numerical invariant to cach right (or left) ideal of $k G$, and possibly this may be of some interest in its own right. It is closely related to the invariant $d$ discussed in [5], but is not quite the same. Our hope was that this invariant would distinguish between essential right ideals and the rest, but we give an cxample indicating the contrary. A result giving a positive answer to the question when $k G$ is regular and $H$ has finite index is proved in [2, Lemma 1.4]. The proof there is rather indirect and it would be interesting to have a direct proof in that case.

Definition 1.1. Let $G$ be a locally finite group, $k$ be a field, and $J$ be a right ideal of $k G$. For each finite subgroup $F$ of $G$, let $\alpha(J, F)=$ $\operatorname{dim}(J \cap k F) /|F|$. Let $\alpha(J)=\sup \alpha(J, F)$, where $F$ ranges over all finite subgroups of $G$.

Of course, the same definition can bc made for left ideals of $k G$. Clearly $\alpha(J) \leq 1$. The main properties of $\alpha(J)$ are the following.

Lemma 1.2. Let $G, k$ be as above and let $H$ be a subgroup of $G$.

(i) If $J$ is a right ideal of $k H$, then $\alpha(J) \leq \alpha(J k G)$.

(ii) If $\alpha(J)=1$, then $J$ is an essential right ideal of $k G$.

(iii) If $\omega(k G)$ denotes the augmentation ideal of $k G$ and $G$ is infinite, then $\alpha(\omega(k G))=1$.

(iv) If $J$ and $L$ are right ideals of $k G$ and $J$ is isomorphic to a submodule of $L$, then $\alpha(J) \leq \alpha(L)$.

These facts clearly imply the following. 
Corollary 1.3. With the above notation, if $H$ is an infinite subgroup of $G$, then $\omega(k H) k G$ is an essential right ideal of $k G$.

This was obtained somewhat less generally in $[6$, p.250] (see below). It is unfortunate that the converse of part (ii) of Lemma 1.2 is false. In fact, we have the following.

Example 1.4. Let $G$ be any countably infinite periodic abelian group. Then for any $\epsilon>0$, there is an essential ideal $J$ of C $G$ such that $\alpha(J)<\epsilon$.

\section{Properties of $\alpha(J)$}

In this section, we shall prove Lemma 1.2 , after mentioning some other basic facts. Throughout, $G$ denotes a locally finite group, and $k$ a field.

Lemma 2.1. Let $J$ be a right ideal of $k G$, and let $F_{1}, F_{2}$ be finite subgroups of $G$ with $F_{1} \leq F_{2}$. Then $\alpha\left(J, F_{1}\right) \leq \alpha\left(J, F_{2}\right)$.

Proof: Clearly, $\left(J \cap k F_{1}\right) k F_{2} \leq J \cap k F_{2}$. Therefore,

$$
\operatorname{dim}\left(J \cap k F_{1}\right)\left|F_{2}\right| /\left|F_{1}\right| \leq \operatorname{dim} J \cap k F_{2} .
$$

Dividing by $\left|F_{2}\right|$ gives the result.

The following is a useful consequence.

Lemma 2.2. Suppose that $G$ is countable, and let $G_{1} \leq G_{2} \leq \ldots$ be a tower of finite subgroups of $G$ such that $\cup_{i=1}^{\infty} G_{i}=G$. Let $J$ be a right ideal of $k G$ and $\alpha_{i}=\operatorname{dim}\left(J \cap k G_{i}\right) /\left|G_{i}\right|$. Then $\alpha(J)=\lim _{i \rightarrow \infty} \alpha_{i}$.

Proof: Let $\beta=\lim _{i \rightarrow \infty} \alpha_{i}$. Clearly, $\beta \leq \alpha(J)$. On the other hand, if $F$ is any finite subgroup of $G$, then $F \leq G_{i}$ for some $i \geq 1$, and then Lemma 2.1 shows that $\alpha(J, F) \leq \alpha_{i} \leq \beta$. Hence $\alpha(J) \leq \beta$, and the two are equal.

Proof of Lemma 1.2: (i) Let $F$ be a finite subgroup of $I I$. Then clearly $J \cap k F \leq J k G \cap k F$. Hence $\operatorname{dim} J \cap k F /|F| \leq \operatorname{dim} J k G \cap k F /|F| \leq$ $\alpha(J k G)$. Since $F$ is an arbitrary finite subgroup of $I$, this gives $\alpha(J) \leq$ $\alpha(J k G)$.

(ii) Let $J$ be a right ideal of $k G$ with $\alpha(J)=1$, and suppose if possible that $L$ is a non-zero right ideal of $k G$ with $J \cap L=0$. Fix a finite subgroup $E$ of $G$ such that $L \cap k E \neq 0$, and let $F$ be any finite subgroup of $G$ containing $E$. Then $(L \cap k E) k F \cap(J \cap k F)=0$, and so $\operatorname{dim}(L \cap$ $k E)|F| /|E|+\operatorname{dim}\left(J \cap k F^{2}\right) \leq|F|$. Hence $\alpha(J, F) \leq 1-\alpha(L, E)<1$. 
Now if $F_{1}$ is any finite subgroup of $G$ and we take $F=\left\langle E, F_{1}\right\rangle$, then we deduce from Lemma 2.1 that $\alpha\left(J, F_{1}\right) \leq 1-\alpha(L, E)<1$, whence $\alpha(J)<1$, a contradiction.

(iii) This is trivial.

(iv) Let $F_{1}$ be any finite subgroup of $G$, and let $\phi: J \rightarrow L$ be a right G-monomorphism. Then $\phi$ maps $J \cap k F_{1}$ into $L \cap k F_{2}$, for some finite subgroup $F_{2}$ of $G$. Let $F=\left\langle F_{1}, F_{2}\right\rangle$. Then $\phi$ embeds $\left(J \cap k F_{1}\right) k F$ into $L \cap$ $k F$. Therefore, $\operatorname{dim}\left(J \cap k F_{1}\right)|F| /\left|F_{1}\right| \leq \operatorname{dim}(L \cap k F)$. Therefore $\alpha\left(J, F_{1}\right) \leq$ $\alpha(L, F) \leq \alpha(L)$, and since $F_{1}$ is arbitrary, the result follows.

We note that if $G$ is a finite elementary abelian 2-group of order $2^{n}$ and $k$ is as field of characteristic 2 , then $\xi^{2}=0$ for all $\xi \in \omega(k G)$. Hence $k G \xi$ annihilates $\xi$, and so $\operatorname{dim} \xi k G \leq 2^{n-1}$. It follows from this that if $H$ is an infinite elementary abelian 2-group, then $\alpha(J) \leq 1 / 2$ for each principal ideal $J \leq \omega(k G)$. This may be compared with [6, p.250].

\section{The Example}

Write $G=\cup_{i=1}^{\infty} G_{i}$, where the $G_{i}$ form a strictly increasing tower of finite subgroups of $G$. We construct the ideal $J$ to satisfy the following condition:

(*). For each $i$ and primitive idempotent $e \in \mathrm{CG}_{i}$, there exists $\alpha \in \mathrm{C} G$ such that $0 \neq$ ea $\in J$.

Since each non-zero ideal of $\mathrm{CG}$ contains such an element $e$, we see that (*) implies that $J$ is essential in $C G$.

Now we construct $J$ as the union of a tower $J_{1} \leq J_{2} \leq \ldots$; where $J_{i}$ is an ideal of $\mathrm{C} G_{i}$. We begin with any minimal ideal of $C G_{1}$ as $J_{1}$. Thus,

$$
\operatorname{dim} J_{1}=1 \text {. }
$$

We also let $e_{1}, e_{2}, \ldots$ be a sequence formed by taking first the (finitely many) primitive idempotents in $C G_{1}$, then those in $C G_{2}$, and so on.

Suppose we have $J_{i}$, a proper ideal of $\mathrm{CG}_{i}$. Then there is a primitive idempotent $e$ of $C G_{i}$ not in $J_{i}$, and if we write $\overline{J_{i}}=J_{i} \mathrm{C} G_{i+1}$, we have the direct sum $e \mathrm{C} G_{i+1} \oplus \overline{J_{i}}$ of ideals of $C G_{i+1}$. Choose the first $j$ such that we have a direct sum $e_{j} \mathrm{C} G_{i+1} \oplus \overline{J_{i}}$. Thus 
For each $l<j$, there exists $\alpha \in \mathrm{CG}_{i+1}$ such that $0 \neq e_{l} \alpha \in \overline{J_{i}}$.

It will also be clcar from the next step that $j \geq i-1$. Let $f$ be a primitive idempotent in $e_{j} \mathrm{C} G_{i+1}$, and put

$$
J_{i+1}=\overline{J_{i}} \oplus \mathrm{C} f,
$$

an ideal of $\mathrm{C} G_{i+1}$. Clearly, $J_{2} \leq J_{i+1}$, and

$$
\operatorname{dim} J_{i+1}=1+\left(\operatorname{dim} J_{i}\right)\left|G_{i+1}\right| /\left|G_{i}\right|
$$

Also, we claim that

$$
J_{2+1} \cap \mathrm{C} G_{i}=J_{i},
$$

from which it follows in particular that $J_{i+1}$ is a proper ideal of $\mathrm{CG}_{i+1}$. To verify the above claim, it suffices to note that if $J_{i+1} \cap \mathrm{C} G_{i}=L_{i}>J_{i}$, then $\operatorname{dim} J_{2+1} \geq\left(\operatorname{dim} L_{i}\right)\left|G_{i+1}\right| /\left|G_{i}\right| \geq \operatorname{dim} J_{i+1}+\left|G_{i+1}\right| /\left|G_{i}\right|-1$, a contradiction, since the sequence $\left(G_{i}\right)$ is strictly increasing. Putting $J=\cup_{i=1}^{\infty} J_{2}$, we have (*), and so $J$ is essential in CG.

Let $\alpha_{i}=\operatorname{dim} J \cap C G_{i} /\left|G_{i}\right|$. Then from (2) and (3), we have $\alpha_{i+1}=$ $\alpha_{i}+1 /\left|G_{i+1}\right|$, so from (1),

$$
\alpha_{i}=\sum_{j=1}^{i} \frac{1}{\left|G_{j}\right|} .
$$

Now $\left|G_{i+1}\right| \geq 2\left|G_{i}\right|$, so

$$
\alpha_{i} \leq \frac{1}{\left|G_{1}\right|}\left(1+\frac{1}{2}+\ldots+\frac{1}{2^{i-1}}\right) \leq \frac{2}{\left|G_{1}\right|} .
$$

Choosing $G_{1}$ suitably, we obtain what we want.

\section{Proof of the Theorem}

The theorem follows from [2, Theorem 1.3] and Lemma 4.1 below.

Lemma 4.1. Suppose that $k G$ is regular and the type I part of $Q^{r}(k G)$ is non-zero. Then $G$ is $\Delta$-hypercentral.

Before beginning the proof we recall some notation and terminology. Let $\Delta(G)=\left\{g \in G:\left|G: C_{G}(g)\right|<\infty\right\}$. We define the transfinite upper $\Delta$-series of $G$ by the rules

$$
\begin{aligned}
\Delta_{0}(G) & =1, \\
\Delta_{p+1}(G) / \Delta_{\rho}(G) & =\Delta\left(G / \Delta_{\rho}(G)\right), \\
\Delta_{\beta}(G) & =\cup_{\alpha<\beta} \Delta_{\alpha}(G),
\end{aligned}
$$


for ordinals $\rho$ and limit ordinals $\beta$. The last term in this series is denoted by $\Delta_{\infty}(G)$ and called the $\Delta$-hypercentre of $G$. We say that $G$ is $\Delta$ hypercentral, if $G=\Delta_{\infty}(G)$.

We also write $\pi(G)$ for the set of primes $p$ such that $G$ has an element of order $p$.

We use implicitly the following fact, which is well known. Since we have not found an explicit reference in the literature, we give a proof for complcteness. Recall that an idempotent $e$ in a regular ring $R$ is called abelian, if every idempotent in $e R e$ is central.

Lemma 4.2. Let $R$ be a regular ring with maximal right quotient ring $Q$. Then $R$ contains a non-zero abelian idempotent if and only if $Q$ does.

Proof: Let $e$ be an abelian idempotent in $Q$. Then $e Q \cap R \neq 0$, so $e Q \cap R$ contains a non-zero idempotent $f$. Now the map $\varphi: f Q f \rightarrow f e Q f e$ defined by $\varphi(x)=x e=e x e$ is a ring isomorphism with invers $\varphi^{-1}(y)=$ $y f$. Since $f e Q f e \subseteq e Q e$, it follows that $f Q f$ is abelian. Therefore so is $f R f$.

Conversely, let $e$ be an abelian idempotent in $R$. We claim that $e R e$ is essential as a right $e R e$-submodule of $e Q e$, whence $[3$, Theorem 3.2 and Corollary 7.4] show that $e Q e$ is abelian. Now $e R=e R e \oplus e R(1-e)$, and since the second summand can contain no non-zero idempotent, it can contain no non-zero right ideal of $R$. Let $x$ be a non-zero element of $e Q e$. Then $x R \cap R$ is a non-zero right ideal of $R$ contained in $e R$, and hence it is not contained in $e R(1-e)$. Therefore there exists an element $r \in R$ such that $0 \neq x r e \in e R e$, and clearly we can replace $r$ by ere here, as required.

Proof of Lemma 4.1: Since the type $I$ part of $Q^{r}(k G)$ is non-zero, $k G$ contains a non-zero abelian idempotent $e$. Let $H$ be the subgroup generated by the support of $e$, and $G_{1}=\left\langle\Delta_{\infty}(G), H\right\rangle$. Then $e$ is an abelian idempotent of $k G_{1}$, and so the type $I$ part of $Q=Q^{r}\left(k G_{1}\right)$ is non-zero. Since $G_{1}$ is $\Delta$-hypercentral, [2, Theorem 1.3] tells us that the type $I_{\infty}$ part of $Q$ is zcro, and therefore its type $I_{f}$ part must be non-zero. By [1, Theorem 2.3], $\left|G_{1}: \Delta\left(G_{1}\right)\right|<\infty$ and $\Delta\left(G_{1}\right)^{\prime}$ is finite. Further, if $M$ is the smallest normal subgroup of $G_{1}$ such that $G_{1} / M$ is abelian-by-finite, then the type $I$ part of $Q$ is $(\hat{M} /|M|) Q$, by the first part of the proof of Theorem 2.3 in [1]. Now $M$ is also the smallest normal subgroup of $\Delta_{\infty}(G)$ such that $\Delta_{\infty}(G) / M$ is abelian-by-finite, and as such, it is normal in $G$. Since $e \in(\hat{M} /|M|) k G \cong k[G / M]$, we see that we may assume that $\Delta_{\infty}(G)$ is abelian-by-finite. By [7, Lemma $12.2 .2 \mathrm{f}, \Delta_{\infty}(G)$ has a characteristic abelian subgroup $A$ of finite index. 
Now $A$ is the direct product $A=\prod A_{p}$ of its primary components. Let $\pi=\pi(H)$, and $A_{\pi^{\prime}}=\prod_{p \notin \pi} A_{p}$. Then $A_{\pi^{\prime}} \mathrm{C} G$. Consider $\vec{G}=G / A_{\pi^{\prime}}$. By $[3$, Lemma 7.6$]$, the image of e in $k \bar{G}$ is an abelian idempotent, and clearly it is non-zero. Therefore the type $I$ part of $Q^{r}(k \bar{G})$ is nonzero. Further, $\Delta_{\infty}(\bar{G})=\Delta_{\infty}(G) / A_{\pi^{\prime}}$. Therefore, we may assume that $A_{\pi^{\prime}}=1$, and so $\pi\left(\Delta_{\infty}(G)\right)=\sigma$ is finite.

Let $p$ be a prime such that $\alpha(e k G)>\frac{1}{p}$, and $p \notin \pi \cup \sigma$. We shall see that $p \notin \pi(G)$. Suppose on the contrary that $G$ contains an element $g$ of order $p$. Let $\vec{g}=1+g+\ldots+g^{p-1}$. Since the powers of $g$ lie in distinct coscts of $G_{1}$, we see that ge $\neq 0$. Since $k G$ is regular, there exists $\beta \in k G$ such that

$$
\tilde{g} e=\bar{g} e \beta \tilde{g} e .
$$

By squaring it, we see that $e \beta \bar{g} e$ is an idempotent in $e k G e$. By $[2$, Lemma. 2.1 , we see that $e \beta \bar{g} e \in k G_{1}$. Using the fact that we have a direct sum $\sum_{l=0}^{p-1} g^{2} k G_{1}$, we deduce from (4) that $e=e \beta \tilde{g} e$. It follows from this that the map $f: e k G \rightarrow \tilde{g} e \beta k G$ defined by $f(\gamma)=\tilde{g} \gamma$ is an isomorphism, $\left(f^{-1}(\delta)=e \beta \delta\right)$, and so by Lemma 1.2 (iv), $\alpha(e k G) \leq \alpha(\tilde{g} k G)$. But an easy calculation shows that $\alpha(\tilde{g} k G)=\frac{1}{p}$. This contradicts the choice of $p$. Hence $\pi(G)$ is finite. By [1, Proposition 1.2], we find that $G$ is hypercentral, and the proof is complete.

\section{References}

1. FERRAN CEOÓ, On the maximal quotient ring of regular group rings, J. Algebra 115 (1988), 164-174.

2. FERRAN Cedó, The maximal quotient ring of regular group rings II, Proc. Amer. Math. Soc. 104 (1988), 357362.

3. K. R. GOODEARL, "von Neumann regular rings," Pitman, Isondon, 1979.

4. J.-M. Goursaud ET J. ValetTe, Sur l'enveloppe injective des anneaux de groupes réguliers, Bull. Soc. Math. France 103 (1975), 91102.

5. J. HANNAH AND K. C. O'MEARA, Maximal quotient rings of prime group algebras, Proc. Amer. Math. Soc. 65 (1977), 1-7.

6. K. C. O'MEARA, Quotient rings of symmetric and universal group algebras, Comm. Algebra 8 (1980), 235-260. 
7. D. S. PASSMAN, "The algebraic structure of group rings," WileyInterscience, New York, 1977.

Ferran Cedó:

Departament de Matemàtiques

Universitat Autonoma de Barcelona

Bellaterra (Barcelona)

SPAIN
Brian Hartley:

Department of Mathematics University of Manchester Manchester M13 9PL

ENGLAND

Rebut el 18 de Desembre de 1991 